\title{
En torno al potencial transformador de los CMS (Critical Management Studies)
}

\author{
On the Transformative Potential of CMS (Critical Management \\ Studies)
}

\author{
ERNESTO GANTMAN
}

Universidad de Buenos Aires y Universidad de Belgrano (Argentina)

Artículo recibido: 24 abril 2016

Solicitud de revisión: 3 junio 2016

Artículo aceptado: 12 octubre 2016

Resumen

El presente ensayo examina los cms en términos de su potencial transformador respecto al mundo del trabajo. A tal efecto, se brinda una caracterización de este enfoque y un repaso de su desarrollo histórico. Se argumenta que los cms no se agotan en su versión británica, disciplinariamente limitada a escuelas de negocios, y que su objeto ha sido también abordado por académicos de diversos países con afiliaciones institucionales en ciencias sociales distintas a la administración. Finalmente, se concluye que la pluralidad disciplinaria de los cмs y el tipo de preocupación intelectual que los motiva permite identificar un rol transformador más afín a disciplinas como la sociología que al campo de la administración. Más que agentes de cambio organizacional, los académicos que trabajan dentro del ámbito de los CMs, podrían convertirse en intelectuales públicos comprometidos con la denuncia de las ideologías gerenciales y económicas dominantes.

Palabras clave: critical management studies, intelectuales públicos, escuelas de negocios, sociología del conocimiento.

\begin{abstract}
This essay examines Critical Management Studies (CMS) in terms of their transformative potential regarding the world of work. To this end, I briefly characterize CMs and review their historical development in the UK. I argue that the main theoretical concerns of CMS are not the exclusive purview of its British version, but that they have also been addressed by scholars from other countries and institutional affiliations in social sciences other than management. Finally, I conclude that the disciplinary plurality of CMS and the intellectual concerns that drive them are clearly aligned with a transformative role more akin to fields like sociology than to management and organization studies. More than organizational change agents, CMs scholars could become public intellectuals committed to unmasking the dominant economic and managerial ideologies.
\end{abstract}

Keywords: critical management studies, public intellectuals, business schools, sociology of knowledge. 


\section{INTRODUCCIÓN}

A partir de la década de los noventa surgió en el Reino Unido una importante corriente de pensamiento denominada Critical Management Studies (CMs) o Estudios Críticos de Gestión, cuya característica central es la aplicación de marcos de referencia de teoría social crítica a los fenómenos organizacionales -entendiendo por «teoría social crítica» ${ }^{1}$ un conjunto extremadamente heterogéneo de enfoques cuyo denominador común es un importante grado de rechazo al orden social capitalista contemporáneo-. En tal sentido, lo que resulta especialmente interesante es que esta corriente de pensamiento ha sido impulsada por académicos con afiliación institucional en escuelas de negocios, organizaciones que a priori no parecen el lugar ideal para albergar la prédica de un discurso indudablemente antitético al mundo actual de los negocios (Fernández Rodríguez, 2007a). Debido a que la vitalidad de los cms sigue firme, el objetivo de este trabajo es ofrecer algunas reflexiones preliminares acerca de sus posibilidades prácticas de alterar el mundo de las organizaciones.

En la primera sección del artículo, precisaremos qué se entiende por CMs, y examinaremos cómo surge y qué dinámica ha facilitado su crecimiento. Entender los factores que han posibilitado su desarrollo nos permitirá extrapolar algunas ideas acerca de su futuro, particularmente en los países en los cuales no ha penetrado demasiado todavía. Al enfrentarnos a un corpus de literatura académica en crecimiento, particularmente si es producido por investigadores sociales que se desempeñan en instituciones cuyo objetivo central es brindar conocimiento «útil», surge el interrogante acerca de su relevancia práctica: ¿pueden cambiar algo los cms? En la segunda sección, intentaremos esbozar algunas respuestas a esta pregunta. Pero no vamos a dejar demasiado en suspenso al lector, ya que contestar el interrogante planteado remite a otro semejante: ¿cuál es el potencial transformador del análisis sociológico crítico? Para responder a ello es pertinente tomar como punto de partida la idea de que el orden social contemporáneo en Occidente está ligado a una auténtica factoría de sentido conformada por un conjunto diverso de discursos que lo legitiman y lo presentan, en diversos grados, como natural, inevitable e incluso deseable (Alonso y Fernández Rodríguez, 2013). Dentro de estos discursos, el subconjunto de aquellos articulados en torno al mundo del trabajo y la economía juega un papel central. El complejo ajedrez de la crítica de los discursos puede pa-

1 Bajo este rótulo consideramos no solo la teoría crítica asociada al círculo de Frankfurt, sino diversos enfoques como el neomarxismo, el postestructuralismo, el postcolonialismo y el feminismo que procuran desvelar diversas formas institucionalizadas de opresión social. 
recer carente de efectos reales, pero autores como Boltanski y Chiapello (1999) consideran que la crítica del capitalismo tiene influencia sobre los propios patrones de desarrollo de este sistema, tanto en términos de sus dispositivos de acumulación como de justicia. Si esta argumentación se considera válida y efectivamente la crítica puede operar sobre el plano real de funcionamiento del capitalismo, así como contribuir a la modificación de sus «espíritus» legitimantes, entonces les cabe un rol a los intelectuales críticos y, por extensión, a los partidarios de los cMs.

\section{SURGIMIENTO Y DESARROLLO DE LOS CMS}

La década de los setenta fue especialmente importante en el desarrollo de los estudios organizacionales. En dichos años se publicaron artículos liminares sobre ecología organizacional, neoinstitucionalismo organizacional y teoría de la dependencia de recursos. Hacia fines de la década hubo llamamientos en favor de la reflexión teórica y la investigación empírica basados en una pluralidad mayor de paradigmas, frente a lo que se percibía como el predominio del enfoque "funcionalista» en ciencias sociales (Burrell y Morgan, 1979). Se comparaba la multiplicidad de paradigmas propia de la sociología con los paradigmas que servían de marco teórico a los estudios organizacionales, entre los cuales la utilización de enfoques críticos basados en la idea de una realidad social caracterizada no por el orden sino por el conflicto, era virtualmente nula. La recepción positiva de esos llamamientos fue paulatina pero sostenida. En los años ochenta, Gareth Morgan (1986) publicó un libro de texto cuyo éxito en términos de difusión indicaba que las posibilidades de apertura paradigmática en los estudios organizacionales estaban abiertas.

El campo de las relaciones industriales y la sociología del trabajo se vieron fuertemente influenciados en el Reino Unido por una obra clásica de Braverman (1974) que generó un intenso debate dando lugar a la teoría del proceso del trabajo. Algunos de los académicos que formarían parte de los cms fueron comentaristas, a favor o en contra, de este enfoque (Rowlinson y Hassard, 2011).Además, distintas vertientes de lo que en teoría social se conoce como postmodernismo influyeron en los teóricos organizacionales británicos y se fue conformando un espacio intelectual en el cual era viable importar teorías y tradiciones de la sociología y la filosofía para la reflexión en torno al fenómeno organizacional. El postestructuralismo, al menos inicialmente, no pareció interesar a los académicos estadouniden- 
ses en estudios organizacionales. Al respecto, en referencia a un artículo enviado en 1984 a Administrative Science Quarterly sobre los aportes de Foucault al estudio de las organizaciones, Gibson Burrell (1996: 652) declaró que conservaba los comentarios de los revisores por cuanto «los tres cuestionaron la relevancia de "un desconocido filósofo francés" y se preguntaron qué "podía una audiencia americana aprender" de su pensamiento». Del otro lado del Atlántico, la recepción de este intelectual fue mucho mayor y se incrementó a lo largo de los años.

En definitiva, creemos que la búsqueda de la novedad junto a un interés más serio por el estudio de la teoría social constituyen factores que explican el desarrollo de lo que luego fueron los cMs en el Reino Unido. En paralelo, se apreciaba un agotamiento de la teoría de la contingencia en los estudios organizacionales. Considerando, además, que la sociología británica, al menos hasta años recientes, pareció siempre privilegiar la investigación cualitativa sobre la cuantitativa (Platt, 2012), era esperable que la reflexión intelectual y las publicaciones de los académicos británicos se fuesen orientando paulatinamente hacia el campo no funcionalista y más explícitamente crítico de la lectura del mundo del trabajo.

Naturalmente, uno de los aspectos que más han resaltado las narrativas del surgimiento de los CMs en el Reino Unido ha sido el proceso de migración de los departamentos de sociología a las escuelas de negocios de los académicos británicos (Martínez Lucio, 2007; Parker, 2015). ${ }^{2}$ En efecto, mientras el presupuesto de los departamentos de sociología se achicaba y las oportunidades de crecimiento profesional en el área disminuían correlativamente, el crecimiento vigoroso de las escuelas de negocios constituía un magneto de atracción para muchos investigadores en ciencias sociales. La expansión de la educación superior en negocios resultó especialmente notable a partir de los años noventa, posiblemente como resultado de la globalización del capitalismo. El Reino Unido no fue la excepción y universidades como Cambridge y Oxford crearon sus escuelas de negocios durantes esos años. Lo que necesitaban especialmente las escuelas de negocios era ganar legitimidad, algo que requería respetabilidad académica, y una manera de lograrlo era contratando académicos de prestigio que pudiesen contribuir con publicaciones en revistas internacionales con referato. Poco

2 Véase especialmente el texto de Martínez Lucio (2007) que comenta cómo factores propios del entorno político y económico del Reino Unido, bajo el gobierno conservador (1979-1997), contribuyeron a fomentar la migración de académicos hacia las escuelas de negocios. Por su parte, Fournier y Grey (2000) señalan el creciente ascenso del managerialismo en dicho país, tanto en el sector privado como en el sector público, como un factor que constituye el telón de fondo para la aparición de la crítica frente a dicho fenómeno. 
importaba a este respecto que el foco de las mismas fuese crítico o no. El ejemplo de la academia británica se contagió rápidamente a países de su área de influencia lingüística o cultural como Australia, Nueva Zelanda y, en menor medida, Canadá. Otras áreas de expansión fueron los países escandinavos (Hartmann et al., 2016). Los Estados Unidos se mantuvieron en cierta medida al margen, a pesar de que las tradiciones críticas en sociología y filosofía eran ampliamente conocidas e incluso cultivadas en sus universidades más prestigiosas (recordemos, por ejemplo, el paso por los Estados Unidos de los teóricos europeos emigrados como Adorno, Horkheimer y Marcuse).

La denominación cms ha sido tomada de una colección de artículos compilada por Alvesson y Willmott (1992) a principios de los noventa. Dichos autores publicaron en 1996 un libro sobre administración que recoge prácticamente el estado del arte de esta perspectiva en su momento. En años posteriores, los principales critters, como se suelen autodenominar los cultores de esta línea académica, aseguraron el crecimiento de los CMS a través de la formación de discípulos en programas doctorales en administración y estudios organizacionales. ${ }^{3}$ Así, la tribu académica de los critters ya no se limitó a los emigrados de otras disciplinas, sino que las escuelas de negocios comenzaron a producir una nueva generación de académicos críticos.

Cabe ahora precisar a qué apuntan los cms y cuál es el sentido del adjetivo «crítico» en este acrónimo. Según Adler, Forbes y Willmott (2007: 120), los CMs tienen por objeto problematizar «los patrones y estructuras socialmente divisivas y ecológicamente destructivas -como el capitalismo, el patriarcado, el imperialismo y similares- que condicionan la acción local y la sabiduría convencional». Por su parte, en una caracterización ampliamente difundida, Fournier y Grey (2000) argumentan que los CMs son marcadamente antiperformativistas, entendiendo el performativismo (performativity) como la búsqueda de un efecto en términos de medios/fines como eficiencia y productividad, aspiraciones centrales en el pensamiento administrativo tradicional. Otra línea demarcatoria respecto a este pensamiento es la «naturalización/desnaturalización». Si la administración tradicional puede concebirse como la construcción social de una realidad particular, el trabajo de los CMs apunta a «desnaturalizar» dicha realidad, a revelarla como una construcción social, histórica y geográficamente con-

3 Los critters constituyen uno de los grupos de interés más numerosos e internacionalizados de la Academy of Management. Uno de los primeros en utilizar el término en trabajos académicos fue Paul Adler (2002). 
tingente. Por último, los CMs son considerados por dichos autores como «reflexivos», en el sentido de que discuten explícitamente sus presupuestos ontológicos y epistemológicos, mientras que la administración tradicional, salvo escasas excepciones, presta una atención muy limitada a sus propios presupuestos.

En síntesis, las formulaciones anteriores nos remiten a un conjunto diverso de teorías, no necesariamente compatibles entre sí, que indagan acerca de la naturaleza del fenómeno organizacional inspiradas en una visión crítica de izquierda, cuyo énfasis está puesto en las asimetrías de poder entre actores y en cómo las mismas influyen sobre los problemas estudiados. De hecho, muchos trabajos de cMs consisten en explicar situaciones particulares de la dinámica organizacional como emergentes de una distribución de poder asimétrica en las organizaciones y el mundo social. El tema del poder resulta así omnipresente en los cMs, que intentan ilustrar cómo las asimetrías de poder generan procesos de discriminación y exclusión de actores en las organizaciones y están sostenidos por discursos legitimantes que construyen identidades instrumentales en la reproducción de las desigualdades.

Fines teóricos de esta índole son abordados desde distintos marcos y tradiciones de las ciencias sociales, a veces con puntos de fricción entre sí, lo cual ha alimentado no pocos debates internos dentro de este enfoque. Lo importante es que los CMs no responden a un marco teórico único, sino a una pluralidad de enfoques cuya característica unificadora es la actitud de denuncia. Por cierto, investigar empíricamente los aspectos negativos de las organizaciones no ha sido monopolio exclusivo de los cms. Por el contrario, temáticas como los conflictos laborales, la discriminación, el mobbing, la corrupción y la falta de ética han sido abordadas extensamente desde el mainstream del pensamiento administrativo y de comportamiento organizacional.

La diferencia radica en que mientras tales enfoques consideran estos problemas o aspectos negativos de las organizaciones como disfuncionales o evitables, los teóricos de los cMs son más pesimistas al respecto y ven la raíz de los males en factores sociales más amplios o estructurales, que no son susceptibles de corregirse mediante el simple voluntarismo porque responden a una problemática generada desde el orden social mismo. Así, las soluciones propuestas por las técnicas de ingeniería social de las organizaciones o los llamamientos en procura de mejorar el nivel ético de los dirigentes son percibidos como ingenuos, simplistas e inconducentes, cuando no directamente como dispositivos ideológicos instrumentales 
para la legitimación del propio sistema social. Este cambio radical de enfoque y su consecuente correlato en el nivel de análisis de la causalidad de los fenómenos organizacionales obedecen precisamente a los marcos teóricos de los que se nutren los académicos de CMs, a partir de los cuales adquiere sentido el adjetivo "crítico», ya que el mismo se aplica, primero y principalmente, a un orden social y se inscribe en una tradición política claramente emancipadora. En palabras de Fourier y Grey, «en un nivel básico, los CMs son un proyecto político en el sentido de que apuntan a desenmascarar las relaciones de poder alrededor de las cuales se teje la vida social y organizacional» (2000: 19$)$.

Aquí radica lo que entendemos como una de las principales posibilidades de expansión del campo de los cms y como un elemento que, a su vez, permite explicar su relativa irrelevancia en las escuelas de negocios no británicas. Para ello, es necesario considerar la formación en grado y posgrado de los investigadores en ciencias sociales de distintos países, así como sus posibilidades de inserción laboral en el ámbito de la educación superior. El punto es que, en muchos países, el tipo de formación en las teorías y paradigmas de los cuales se nutren los cMs no es recibida por los universitarios que estudian carreras de ciencias económicas, sino por quienes siguen carreras humanísticas o de otras ciencias sociales. Si bien los estudiantes de las disciplinas que conforman las ciencias económicas y de gestión (economía, contabilidad, auditoría, marketing, administración, etc.) están expuestos a los contenidos de asignaturas introductorias sobre sociología y teoría social, las mismas típicamente ofrecen un tratamiento superficial de su objeto que muchas veces está disociado del resto de la carrera. En términos de conocimiento de gestión, los objetivos pedagógicos que persiguen los estudios apuntan a dotar al estudiante de una formación profesionalista, lo que implica un saber centrado en técnicas y herramientas útiles para el desarrollo de las actividades propias del mundo de las empresas y los negocios. Y los estudios de posgrado en administración, al menos en las maestrías de corte profesionalista, no escapan a esta lógica. ${ }^{4}$ En cambio, quienes estudian carreras humanísticas, sociología u otras ciencias sociales efectivamente adquieren una formación de base más sólida en los marcos teóricos de los cuales se nutren los critters.

4 Quienes intentan especializarse en estudios organizacionales a nivel doctoral tienen posibilidades de estudiar diversas perspectivas de las ciencias sociales, pero hasta ese nivel la exposición de los estudiantes a teorías sociales, especialmente aquellas propias de una tradición crítica del orden social, suele ser bastante reducida. 
Por otra parte, una extensa literatura sugiere que los intelectuales, particularmente aquellos que se dedican a las humanidades y ciencias sociales, tienen una orientación política más afín a la izquierda (Gouldner, 1979; Boudon, 2004; King y Szelényi, 2004). Si los estudiantes de tales carreras que desean dedicarse a tareas de investigación académica eligen como objeto analítico el mundo del trabajo, el resultado casi lógico será un académico que produzca cMs, aunque no lo haga necesariamente bajo este nombre. Incluso las connotaciones mismas del término management le pueden resultar antipáticas y, por supuesto, podrá ignorar muchos de los textos liminares de los cms británicos. Pero el producto de su trabajo será, casi inevitablemente, compatible con el de los critters ingleses (solo las referencias bibliográficas serán distintas, excepto desde luego las citas a autores canónicos como Marx, Habermas o Foucault). En síntesis, mientras que las escuelas de negocios no resultan en muchos países un ámbito muy propicio para el desarrollo de los CMs, existe en ellos un verdadero semillero del que puede surgir una nueva generación de teóricos de cms en departamentos de sociología, ciencias políticas y disciplina afines.

No pretendemos con esto afirmar que la combinación de amplia formación en teoría social y afiliación a escuelas de negocios o facultades de ciencias académicas sea absolutamente inusual. De hecho, en un país en desarrollo como Brasil, dicha combinación caracterizó en los años setenta a algunos académicos pioneros de las perspectivas críticas en administración como Mauricio Tragtenberg y Fernando Prestes Motta, pero se trata de casos aislados. Lo que creemos es que hay potencial humano para el desarrollo de los cms en muchos países, pero no necesariamente desde el locus institucional de su producción en el Reino Unido.Al respecto, y a título de ejemplificación no taxativa, son pertinentes los casos de Israel (Frenkel, 2016) y de varios países sudamericanos (Gantman, 2016). Por otra parte, incluso en países con una destacada tradición crítica en ciencias sociales, como Francia, los cMs solo han comenzado recientemente a ganar partidarios en las universidades y escuelas de negocios (Huault y Perret, 2016), mientras que las perspectivas críticas sobre el mundo del trabajo tenían desde hace muchos años singular vitalidad en la comunidad académica de la sociología. Otro ejemplo es España, donde con algún retraso se asiste a la introducción paulatina de los CMs pero desde la sociología y no el campo de los estudios en gestión (Fernández Rodríguez, 2007b). En China y Japón, los CMs no han tenido relevancia para los académicos en administración, a pesar de que las perspectivas críticas y el marxismo tienen una singular 
presencia entre los científicos sociales, como señala Kiyomiya (2016) para el caso japonés.

Esto no debe ser percibido como un problema, sino como una oportunidad, que sin embargo requiere una extensión de nuestro propio concepto de cms. Así, la M (management) del acrónimo podría ser reemplazada por una $\mathrm{O}$ de organización. Podríamos hablar perfectamente de ECO (estudios críticos organizacionales), ya que como dijimos, la palabra management tiene connotaciones gerencialistas que remiten a lo normativo y, por lo tanto, pueden suponer un énfasis que resulta ajeno o indeseado para los académicos provenientes de otras disciplinas. Además, tal como lo demuestran los trabajos recogidos en el libro de Grey, Huault, Perret y Taskin (2016), existe una polifonía de voces globales que se ocupan de temas propios de los CMs sin necesariamente mantener vínculos estrechos a nivel de red de citas académicas con la vertiente británica de los CMs. Provincializar los CMs, no solo geográficamente en el Reino Unido sino a nivel de las escuelas de negocios, expandiendo las fronteras disciplinarias (Delbridge, 2014), es perfectamente posible. En rigor, podría incluso ser deseable en términos del futuro desarrollo de esta tradición académica, cuya internacionalización sin depender de un centro es perfectamente compatible con sus propias premisas axiológicas. Autores como Prasad, Prasad, Mills y Mills (2016) han expresado una perspectiva similar para su visión futura de los CMs, a partir de la superación de los límites impuestos por lo que denominan la escuela de Manchester en CMs y de una apertura epistemológica hacia problemáticas y perspectivas no occidentales (Alcadipani y Rosa, 2011; Alcadipani et al., 2012; Faria, 2013; Prasad, 2016).

\section{CMS: ¿DE LA DENUNCIA A LA PROTESTA?}

Hemos sugerido en la sección anterior que los cms tienen un interesante potencial de desarrollo futuro, especialmente si los concebimos en un sentido amplio que excede, por un lado, su vertiente británica y, por el otro, la estrecha identificación institucional con las escuelas de negocios. Ahora bien, volviendo nuevamente al "grupo duro» de los cms en el Reino Unido, es pertinente preguntarnos qué tipo de conocimiento generan. Examinar rigurosamente esta cuestión demandaría un extenso estudio bibliométrico, pero en términos de categorías generales podemos plantear dos grandes categorías: $a$ ) análisis de prácticas y situaciones organizacionales concretas, y b) análisis de los discursos que representan dichas prácticas y even- 
tualmente influyen sobre ellas. ${ }^{5}$ Ambos aspectos están naturalmente muy imbricados, ya que los discursos pueden legitimar prácticas pero también contribuir a originarlas.Además, las prácticas existentes pueden servir, a su vez, para inspirar los discursos de las ideologías gerenciales que las legitiman y contribuyen a su reproducción en el mundo del trabajo. Lo que une este vasto conjunto de producción académica es la denuncia de asimetrías de poder como factor último explicativo de los problemas o situaciones indeseables que se detectan (alienación, explotación, discriminación, etc.). El desafío es pasar del nivel descriptivo, la denuncia, al nivel propositivo. Esto, naturalmente, nos lleva al terreno de la performatividad crítica, ya que, como señala Martin Parker (2002), acabar con la explotación tiene en última instancia un claro sentido performativo. De acuerdo con Spicer, Alvesson y Kärreman (2009), los académicos de CMS no deben contentarse solo con entender la realidad, sino que tienen que preocuparse por cambiarla. Si la emancipación es un objetivo al que aspiran dichos académicos, el cambio deseable es generalmente interpretado en términos de dos conceptos: la macroemancipación, que remite a cambios al nivel de orden social, y la microemancipación, que simplemente se refiere a cambios menores, incluso temporarios, en las prácticas cotidianas del mundo laboral (Huault et al., 2016). ${ }^{6}$ La primera de ellas es generalmente percibida como un objetivo carente de realismo (Rowlinson y Hassard, 2011), mientras que la microemancipación es considerada como algo perfectamente realizable. En la búsqueda de la emancipación, se ha propuesto la noción de performatividad crítica, que «involucra una intervención activa y subversiva en los discursos y prácticas gerenciales» (Spicer et al., 2009: 538).

Este llamamiento de algunos académicos en favor de fomentar el involucramiento con la práctica en organizaciones reales surge frente a la insatisfacción con lo que perciben como una postura excesivamente teórica de los CMs (Spicer et al., 2009; Wickert y Schaefer, 2015). El debate, no obstante, se ha traducido en trabajos teóricos más preocupados por desentrañar en forma analítica el sentido de la "performatividad crítica» (Cabantous et al., 2016) que en experimentar al estilo de la investigación-acción en organiza-

5 Podría considerarse también una tercera categoría, el análisis y debate teóricoconceptual sobre el fenómeno organizacional, pero la dejaremos de lado por cuanto la producción teórica de los cMs, en mayor o menor medida, siempre hace referencia a las prácticas y discursos gerenciales.

6 Es útil presentar algunos ejemplos para precisar mejor qué entienden los critters por microemancipación. El humor, el cinismo, el sabotaje frente a las pretensiones de la dirección son formas de microemancipación. Identificar puntos ciegos en el panóptico del control gerencial que posibiliten un espacio, aunque sea temporario o ambiguo, de libertad para el trabajador es un modo de lograr una microemancipación (Alvesson y Willmott, 1996). Ocuparse de actividades privadas en el trabajo es un acto de microemancipación (Huault et al., 2016). 
ciones reales. Una de las excepciones a ello es un reciente trabajo de King (2014) en el cual el autor se involucra en cuatro experiencias empíricas y confiesa que, si bien la perspectiva crítica puede brindarle una óptica distinta para leer la práctica organizacional, esta no le ofrece herramientas para actuar. Este vacío en términos de repertorios de elementos normativos le hizo difícil "como profesional individual, saber cómo hacer las cosas de otro modo» (King, 2015: 262). Por otra parte, algunos críticos de las propuestas orientadas a la performatividad crítica argumentan lisa y llanamente que convertir los cMs en una versión contemporánea del progresismo en investigación-acción no es la vía más adecuada para lograr el cambio social (Spoelstra y Svensson, 2016). ${ }^{7}$ De acuerdo con ellos, los CMs deben limitarse al esclarecimiento intelectual, al desenmascaramiento de los discursos dominantes, «sin necesariamente señalar nuevas soluciones a lo que es concebido como problemático y disfuncional» (Spoelstra y Svensson, 2016: 75). En definitiva, no hay en los trabajos de los cMs un claro modelo normativo de cambio organizacional, solo la búsqueda de un horizonte emancipatorio a partir de actividades de esclarecimiento que se basan en la denuncia de los discursos y prácticas gerenciales dominantes.

No obstante, muchos partidarios de los CMs tienen como utopía el logro de cambios radicales en el funcionamiento de las organizaciones, pero este objetivo se presenta como complicado en el contexto de lo que Martin Parker caracteriza como «atrofia contemporánea de ideas sobre alternativas» (2002: 211). Así, dicho autor plantea la urgente necesidad de alentar la imaginación para concebir formas alternativas de organización, algo a lo cual ha contribuido en un reciente volumen colectivo (Parker et al., 2014).

En nuestra opinión, este es un camino válido para profundizar en los CMS, pero prestando mayor atención analítica a las condiciones materiales y simbólicas de posibilidad de emergencia de tales formas organizacionales alternativas. No es lo mismo pensar la economía solidaria en el contexto de un país del tercer mundo en bancarrota que en el de un país desarrollado. Tampoco concebir y fomentar las organizaciones del tercer sector en una economía próspera que en otra cuya gran parte de la población tiene sus necesidades básicas insatisfechas. El estudio de las formas organizacionales requiere un énfasis importante en el nivel analítico macro. Resulta así

7 Pese al llamamiento de los partidarios de la performatividad crítica, los cMs no se caracterizan por un énfasis en la gestión aplicada y en las intervenciones organizacionales. De allí su diferencia central con enfoques como la investigación-acción, algunos de cuyos partidarios como el noruego Einar Thorsrud (1978) desarrollaron enfoques progresistas del cambio organizacional que no parecían demasiado alejados de las preocupaciones de los cMs, o la sociología clínica, que hace del fuerte involucramiento en las intervenciones sociales un elemento central (De Gaulejac, 2008). 
indispensable la apertura de los cms hacia el campo de la sociología, la economía y la ciencia política, disciplinas donde creemos que puede encontrar un campo de reclutamiento de intelectuales interesados. En definitiva, si los problemas que detectan los partidarios de los CMs suponen un factor último de determinación en el orden social, ninguna solución a nivel organizacional será efectiva si no hay un cambio social más amplio. Esto nos remite a las ciencias sociales que se ocupan de tales factores macro y a disciplinas como las mencionadas anteriormente.

Por otra parte, si la denuncia y la iluminación de la «oscuridad de las prácticas contemporáneas de gestión, la sociedad de consumo y el capitalismo» (Spoelstta y Svensson, 2016: 75) son un objetivo de los critters, entonces deberían buscar una audiencia más amplia, lo que es una invitación para convertirse en intelectuales públicos (Smith-Lovin, 2007; Parker, 2013; Fleming y Banerjee, 2015). ${ }^{8}$ En una obra verdaderamente destacable, uno de los principales exponentes de los cMs, Martin Parker (2002) advierte claramente de esta posibilidad, que en nuestra opinión es un corolario lógico de la naturaleza misma del trabajo intelectual encarado por algunos representantes de los CMs. Si bien dicho autor considera que los académicos pueden contribuir de forma algo limitada al ambicioso objetivo de construir un mundo mejor, sugiere que «las demandas de enseñanza, investigación y tareas administrativas implican que sus empleos e identidades operan en contra de cualquier intento sistemático de convertirse en intelectuales críticos» (Parker, 2002: 132).

Un planteo de Parker (2002: 132) ilustra la locación paradójica de los partidarios de los CMs, al indicar que el éxito de la formación en cMs podría medirse, por ejemplo, en términos de cuántos graduados prefieren trabajar en cooperativas en lugar de aceptar puestos de gerentes de empresas, agregando con singular y demoledora ironía: «cuando las escuelas de negocios se encuentren vacías, cuando sus corredores contengan hojas muertas y sus tejados goteen, entonces serán convertidas en departamentos de sociología o asilos de ancianos, y los cмs habrán hecho su trabajo». Pero el humor irreverente de Parker no deja de tener su costado relevante, ya que la lógica del trabajo analítico de los cMs remite a aspectos sociales amplios, que son precisamente el dominio de la sociología crítica. Como él mismo admite, la aspiración de los critters de llegar a un público más amplio que la reducida comunidad académica o su propia tribu intelectual supone situarse en el rol del intelectual público. Si los CMs aspiran a intentar transformar

8 Russell Jacoby define a los intelectuales públicos como «escritores y pensadores que se dirigen a una audiencia general e instruida» (2000: 5). 
algo en el entorno social, su tarea implicará necesariamente transmitir sus denuncias y, si existieran, sus propuestas de reformas a una audiencia no académica. Bridgman y Stephens sostienen una idéntica opinión, señalando que los cms deberían involucrarse de forma más activa en una audiencia mayor, lo que los llevaría «más cerca de cumplir el potencial emancipatorio de los CMS» (2008: 269). Obviamente, esto implica descender, aunque sea por un tiempo, de la torre de marfil e involucrarse más en el terreno de la sociología, lo cual no debería ser difícil ya que, para muchos, esa es su formación académica de origen.

Por último, si el potencial transformador de los CMs radica en convertirse en intelectuales públicos, la pregunta deviene entonces ¿cuál es la significatividad de los intelectuales públicos en términos de su capacidad de producir un cambio social efectivo??

Cuando un científico social se convierte en intelectual público e intenta influir sobre temas de la agenda pública se transforma en actor político. Cabe recordar en este sentido la importancia del rol de los intelectuales orgánicos en el pensamiento gramsciano. Específicamente en el caso del management, muchos autores de best sellers en la disciplina, algunos de ellos académicos de prestigio, son en cierto sentido intelectuales orgánicos de un discurso que opera para proveer justificaciones argumentales a favor del orden capitalista dominante. Al respecto, en su extenso y celebrado estudio sobre la ideología gerencial contemporánea, Boltanski y Chiapello (1999) otorgan al pensamiento gerencial de moda una importancia simbólica descomunal al asimilarlo a una suerte de espíritu del capitalismo contemporáneo.

Si efectivamente los discursos dominantes contribuyen a construir la realidad y a otorgarle el sentido de lo inevitable o, incluso, lo justo, desenmascararlos es una función para nada trivial de los intelectuales públicos que tienen una actitud crítica ante el orden social. Dicha tarea resulta, entonces, el papel lógico que los partidarios de los CMs deberían aspirar a desempeñar. Recientemente, algunos de ellos ya lo han hecho, ingresando de lleno en la arena de la literatura crítica destinada a una audiencia más amplia. Bajo el nombre grupal de Corporate Reform Collective (2014), ocho académicos, entre ellos importantes figuras de los CMs como Martin

9 Las dudas sobre la capacidad de los intelectuales públicos de coadyuvar al cambio social se extienden también a los especialistas. De hecho, algunos autores han cuestionado la validez de las ciencias sociales, críticas o no, para alterar la realidad social más allá de su pretendida contribución a orientar políticas públicas. Smith, por ejemplo, considera que los recursos económicos e intelectuales destinados a la investigación en ciencias sociales «no han logrado que nuestra política sea apreciablemente más racional» (1993: XII). 
Parker y Hugh Willmott, han publicado una obra que hace un claro llamamiento a favor de acciones del Estado sobre leyes corporativas, políticas financieras, etc. Un paso que puede ser un prolegómeno de un mayor involucramiento práctico de los critters en torno a los debates sobre las políticas públicas en materia de la gobernanza del capitalismo contemporáneo.

Hay que reconocer, no obstante, que la agenda pública no cambia fácilmente y que es necesario ejercer una influencia efectiva sobre los partidos políticos y la opinión pública para lograr efectos en este sentido. ${ }^{10} \mathrm{El}$ propio Parker destaca que «la enseñanza universitaria y la escritura académica son "políticas", pero bastante limitadas en su impacto» (2013: 179), lo cual impone un límite al optimismo ingenuo. La alternativa, no obstante, es la inacción. Según Parker, los CMs «vociferan fuertemente en favor de la emancipación, pero están enclaustrados en puestos de trabajo bien pagados en flamantes escuelas de negocios» (2013: 168). En la medida en que los CMS trasciendan su versión británica, las posibilidades de expansión de este movimiento académico, como lo conceptualiza Willmott (2013) pueden involucrar también renovadas expectativas de intervención en debates interdisciplinarios más amplios y, eventualmente, un rol más productivo desde el punto de vista de la agencia de los intelectuales públicos.

\section{CONCLUSIÓN}

Este trabajo se ha propuesto reflexionar en torno al potencial transformador de los CMs. Primeramente, hemos caracterizado esta perspectiva académica y repasado brevemente su surgimiento en el Reino Unido. En el presente los CMs gozan de muy buena salud en dicho país y han configurado una singular y, en algún punto, contradictoria simbiosis de académicos críticos con escuelas de negocios. Las escuelas de negocios británicas, como en otras latitudes, venden como producto central capacitación útil para los futuros gerentes y líderes de empresas, que muchas veces las financian de forma generosa, pero constituyen también un refugio para intelectuales críticos que contribuyen a incrementar su prestigio académico a través de publicaciones en revistas científicas con referato, algo considera-

10 Por otra parte, y en términos de la eficacia del pensamiento crítico para producir efectos emancipatorios, Boltanski y Chiapello (1999) sugieren que el orden capitalista puede utilizar algunos argumentos formulados por sus críticos para generar nuevos y más sofisticados mecanismos de sujeción de los trabajadores. 
do favorablemente tanto en los ejercicios de evaluación del sistema de educación superior como en los rankings académicos.

En ese contexto, es poco importante que los critters denuncien, con mayor o menor grado de ensañamiento, al sistema económico y social al cual las escuelas de negocios presuntamente sirven. Por otra parte, hemos argumentado que en otros países, tanto del centro como de la periferia, la reflexión y la producción intelectual en temáticas propias de los cMs se da mayormente fuera del contexto institucional de las escuelas de negocios. Vemos allí un enorme potencial de expansión para los CMs, que podrían perfectamente extender su ámbito más allá del reducido espacio original de su vertiente británica.

Nos preguntamos entonces, dada la vitalidad de esta corriente de pensamiento en materia de estudios organizacionales, cuál es su potencial transformador. Es decir, más allá de su obvio énfasis en la denuncia del orden existente, aspecto que lógicamente comparte con las tradiciones intelectuales de las cuales se nutre, nos interesamos en reflexionar acerca de sus perspectivas de transformación social. Una contribución en tal sentido es algo lógicamente esperable, ya que por ser la gestión su objeto disciplinario cabría pensar en algún contenido o lección propositiva. Como respuesta, y teniendo en cuenta que el tema de la performatividad crítica comienza a ser debatido por sus partidarios, entendemos que la máxima potencialidad de los смs radica en dos aspectos básicos, que cabe distinguir analíticamente pero que están en gran medida interrelacionados.

En primer lugar, el campo casi utópico de las formas alternativas de organización en las cuales la coordinación del trabajo no se vea sometida a la lógica del managerialismo tan criticada por distintos autores (Parker, 2002; Klikauer, 2013). En segundo lugar, el campo de la denuncia del orden social, elemento central y casi definicional de los cMs, pero no desde el lugar del académico, cuya audiencia se limita estrictamente a pares y discípulos en productos de limitada difusión que solo contribuyen a validar su prestigio y legitimar su carrera en el ámbito de la educación superior, sino en la construcción de una audiencia mayor como intelectual público, desempeñando un rol contestatario ante las ideologías gerenciales y económicas dominantes. Aunque esta función no necesariamente puede resultar de interés para muchos critters que tal vez prefieran focalizar su esfuerzo intelectual en el debate escolástico, creemos que podría ser atractiva para los nuevos partidarios que la expansión del movimiento encuentre en otras ciencias sociales. Extrañamente, el futuro de los cms, si estos persiguen una búsqueda más activa del cambio social, podría involucrar un regreso disci- 
plinario a sus orígenes. Se trata, desde luego, de un aporte modesto y en principio alejado de las grandes transformaciones sociales, especialmente dada la demostrada plasticidad del orden capitalista para adaptarse frente al pensamiento crítico (Boltanski y Chiapello, 1999), pero en última instancia es la misma tarea que atañe a los pensadores progresistas comprometidos con el cambio social que juzgan deseable.

\section{BIBLIOGRAFÍA}

AdLER, P. (2002): «Critical in the Name of Whom and What», Organization, 9(3), pp. 387-395.

Adler, P., Forbes, L. y Willmott, H. (2007): «Critical Management Studies», Academy of Management Annals, 1(1), pp. 119-179.

AlCADIPANI, R. y Rosa,A. R. (2011): «From global management to glocal management: Latin American perspectives as a counter-dominant management epistemology», Canadian Journal of Administrative Sciences, 28(4), pp. 453-466.

Alcadipani, R., Rafi, F., Gantman, E. y Nkomo, S. (2012): «Southern voices in management and organization knowledge», Organization, 19(2), pp. 131-143.

Alonso, L. E. y Fernández Rodríguez, C. J. (2013): Los discursos del presente. Un análisis de los imaginarios sociales contemporáneos, Madrid, Siglo XXI.

Alvesson, M. y Willmott, H. (eds.) (1992): Critical Management Studies, Londres, Sage.

Boltanski, L. y Chiapello, E. (1999): Le nouvel esprit du capitalisme, París, Gallimard.

Boudon, R. (2004): Pourquoi les intellectuels n'aiment pas le libéralisme, París, Odile Jacob.

Braverman, H. (1974): Labour and Monopoly Capital, Nueva York, Monthly Review Press.

BRIDGMAN, T. y STEPHENS, M. (2008): «Institutionalizing critique: A problem of Critical Management Studies», Ephemera, 8(3), pp. 258-270.

Burrell, G. (1996): «Normal Science, Paradigms, Metaphors, Discourses and Genealogies of Analysis», en Clegg, S. R., Hardy, C., LaWrence, T. y Nord, W. R. (eds.) (1996): Handbook of Organization Studies, Thousand Oaks, Sage. 
Burrell, G. y Morgan, G. (1979): Sociological Paradigms and Organizational Analysis, Londres, Heinemann.

Cabantous, L., Gond, J. P., Harding, N. y Learmonth, M. (2016): «Reconsidering critical performativity», Human Relations, 69(2), pp. 197-213.

Corporate Reform Collective (2014): Fighting Corporate Abus: Beyond Predatory Capitalism, Londres, Pluto Press.

De GaUlejac, V. (2008): Las fuentes de la vergüenza, Madrid, Mármol-Izquierdo Editores.

Delbridge, R. (2014): «Promising Futures: cms, Post-Disciplinarity, and the New Public Social Science", Journal of Management Studies, 51(1), pp. 95-117.

FARIA, A. (2013): «Border Thinking in Action: Should Critical Management Studies Get Anything Done?», en Malin, V., Murphy, J. y Siltaoja, M. (eds.) (2013): Getting Things Done, Bingley, Emerald.

Fernández Rodríguez, C. J. (2007a): «Postmodernidad y teoría crítica de la empresa. Una presentación de los Critical Management Studies», en FerNÁNDEZ Rodríguez, C. J. (ed.) (2007): Vigilar y organizar. Una introducción a los Critical Management Studies, Madrid, Siglo xxI.

- (2007b): «Estudios críticos de la gestión: una visión general», en FERNÁNDEZ RodRíguez, C. J. (ed.) (2007): Vigilar y organizar. Una introducción a los Critical Management Studies, Madrid, Siglo xxI.

Fleming, P. y BANERJEe, S. B. (2015): «When performativity fails: Implications for Critical Management Studies», Human Relations, 69(2), pp. 257-276.

Fournier,V.y Grey, C. (2000): «At the Critical Moment: Conditions and Prospects for Critical Management Studies», Human Relations, 53(1), pp. 7-32.

FrenKel, M. (2016): «From anti-managerialism to over-managerialism: How Critical Management Studies in Israel were exiled from local business schools and emerged elsewhere», en Grey, C., Huault, I., Perret, V. y Taskin, L. (eds.) (2016): Critical Management Studies. Global Voices, Local Accents, London, Routledge.

GanTMAN, E. R. (2016): "CMS in the periphery: A look at South America», en Grey, C., Huault, I., Perret, V., y Taskin, L. (eds.) (2016): Critical Management Studies. Global Voices, Local Accents, Londres, Routledge.

GOULDNER, A. (1979): The Future of Intellectuals and the Rise of the New Class, Londres, Macmillan.

Grey, C., Huault, I., Perret, V. y Taskin, L. (eds.) (2016): Critical Management Studies. Global Voices, Local Accents, London, Routledge.

Hartmann, R., Kärreman, D. y Alvessom, M. (2016): «CMs in Scandinavia: A hitchhiker's guide to the realm of the scandocrits», en Grey, C., Huault, 
I., Perret, V. y Taskin, L. (eds.) (2016): Critical Management Studies. Global Voices, Local Accents, Londres, Routledge.

Huault, I. y Perret, V. (2016): «The awakening of Critical Management Studies in France: Mimicry or a process of coming out?», en Grey, C., Huault, I., Perret, V. y Taskin, L. (eds.) (2016): Critical Management Studies. Global Voices, Local Accents, Londres, Routledge.

Jacoвy, R. (2000): The Last Intellectuals: American Culture in The Age of Academe, New York, Basic Books.

KING, D. (2015): "The possibilities and perils of critical performativity: Learning from four case studies», Scandinavian Journal of Management, 31(2), pp. 255-265.

KING, L. y SZELÉNYI, I. (2004): Theories of the New Class. Intellectuals and Power, Minneapolis, University of Minnesota Press.

Kiуомгун, T. (2016): «Self problematization and relational problematization: A critical constructive approach in the Japanese context», en GreY, C., Huault, I., Perret, V. y Taskin, L. (eds.) (2016): Critical Management Studies. Global Voices, Local Accents, Londres, Routledge.

KLIKAUER,T. (2013): Managerialism.A Critique of an Ideology, Basingstoke, Palgrave.

Martínez Lucio, M. (2007): «¿Neoliberalismo y neoconservadurismo interrumpido? El porqué de la existencia de una tradición crítica en las escuelas de dirección de empresas británicas», en FERnández RodríGuez, C. J. (ed.) (2007): Vigilar y organizar. Una introducción a los Critical Management Studies, Madrid, Siglo xxI.

Morgan, G. (1986): Images of Organization, Beverly Hills, Sage.

Parker, M. (2002): Against Management. Organisation in the Age of Managerialism, Cambridge, Polity Press.

- (2013): "What is to be Done?' CMs as a Political Party» en Malin, V., MurPhy, J. y Siltaoja, M. (eds.) (2013): Getting Things Done, Bingley, Emerald.

- (2015): «Between sociology and the business school: critical studies of work, employment and organization in the UK", The Sociological Review, 63(1), pp. 162-180.

Parker, M., Cheney, G., Fournier, V. y Land, C., (eds.) (2014): The Routledge Companion to Alternative Organization, Londres, Routledge.

PlatT, J. (2012): «Making them count: How effective has official encouragement of quantitative methods been in British sociology?», Current Sociology, 60(5), pp. 690-704. 
PRASAD, A. (2016): «Towards decolonizing modern Western structures of knowledge: A postcolonial interrogation of (Critical) Management Studies», en Prasad, A., Prasad, P., Mills, A. J. y Mills, J. H. (eds.) (2016): The Routledge Companion to Critical Management Studies, Londres, Routledge.

Prasad, A., Prasad, P., Mills, A. J. y Mills, J. H. (2016): «Debating knowledge. Rethinking Critical Management Studies in a changing world", en PRASAD, A., Prasad, P., Mills, A. J. y Mills, J. H. (eds.) (2016): The Routledge Companion to Critical Management Studies, Londres, Routledge.

Rowlinson, M. y Hassard, J. (2011): «How come the critters came to be teaching in business schools? Contradictions in the institutionalisation of critical management studies», Organization, 18(5), pp. 673-689.

Sмгтн, J.A. (1993): The Idea Brokers: Think Tanks and the Rise of the New Policy Elite, Nueva York, The Free Press.

Smith-Lovin, L. (2007): «Do we need a public sociology? It depends on what you mean by sociology", en Clawson, D., Zussman, R., Misra, J., Gerstel, N. y STOKеs, R. (eds.) (2007): Public Sociology: Fifteen Eminent Sociologists Debate Politics and the Profession in the Twenty-first Century, Berkeley, University of California Press.

Spicer, A., Alvesson, M. y Kärreman, D. (2009): «Critical performativity: The unfinished business of critical management studies», Human Relations, 62(4), pp. 537-560.

Spoelstra, S. y P. Svensson, P. (2016): «Critical performativity. The happy end of Critical Management Studies?», en Prasad, A., Prasad, P., Mills, A. J. y Mitls, J. H. (eds.) (2016): The Routledge Companion to Critical Management Studies, Londres, Routledge.

THORSRUD, E. (1978): «Democracy at work and perspectives on the quality of working life in Scandinavia», International Studies of Management \& Organization, 8(1/2), pp. 59-81.

Wickert, C. y SCHEFER, S. (2015): «Towards a progressive understanding of performativity in critical management studies», Human Relations, 68(1), pp. 107-130.

WiLlmotт, H. (2013): «Changing Institutions: Critical Management Studies as a Social Movement», en Malin, V., Murphy, J. y Siltaoja, M. (eds.) (2013): Getting Things Done, Bingley, Emerald. 